\title{
INVESTIGATION OF ARGON PLASMA JETS WITH SPECTRAL LINE INTENSITY RATIO
}

\author{
Murat TANIŞLI ${ }^{1, *}$, Sevinç YANIK ${ }^{1}$, Neslihan ŞAHİN ${ }^{1}$ \\ ${ }^{1}$ Department of Physics, Faculty of Science, Eskişehir Technical University, Eskişehir, Turkey
}

\begin{abstract}
At the atmospheric pressure, argon plasma jet was obtained for $20 \mathrm{kHz}$ frequency, $8 \mathrm{kV}$ voltage and different flow rate in the DBD-like system. The optical emission spectra of these jets were taken. Spectral lines of discharges experimentally obtained were compared with spectral lines in NIST (National Institute of Standards and Technology) database. Atmospheric pressure plasmas are optically thin. Therefore, the line intensity ratio method can be used by determining suitable spectral lines. In general, the intensity of a spectral line is a function of both electron temperature and density. While determining these spectral lines, those sensitive to electron temperature were selected. The spectral lines, which are sensitive to electron temperature, depend on the difference between the excitation energy levels and the transition probability ratios. As a result, the electron temperature of argon jet was tried to be determined by line intensity-ratio method.
\end{abstract}

Keywords: Atmospheric plasma jet, Line intensity ratio method, Electron temperature

\section{INTRODUCTION}

Under laboratory conditions, atmospheric pressure plasmas are more preferred than low pressure plasmas. One of the reasons for this is low cost. In low pressure plasma systems, vacuum system is needed to decrease the pressure value. Therefore, the equipment required for the discharge system is extra cost. The other reason is that the use of atmospheric pressure plasma is more useful than the lowpressure plasma system. For these reasons, it is used quite easily in various fields such as surface treatment of materials, sterilization of instruments in medicine, ineffective rendering of bacteria, and also sterilization of foods [1-3].

There are four different methods to determine the electron temperature and electron density for the plasma at low densities $\left(n_{e}<10^{11} \mathrm{~cm}^{-3}\right)$. These are continuous-radiation analysis, Thomson scattering, Langmuir probe or optical emission spectroscopy (OES) [4]. The density of a spectral line is defined as a function of both electron temperature and electron density [5]. One of the methods used to determine the electron temperature is the line intensity ratio method. This method is obtained by ratio of the intensity values of the two spectral lines selected from the obtained spectrum.

In order to use the line intensity ratio method, the plasma system must be under local thermodynamic equilibrium (LTE) conditions and be optically thin. In little or no scattering condition, the plasma is optically thin. Atmospheric pressure plasmas are also optically thin [6]. Therefore, the ratio of optical emission spectral lines is given by the following equation as

$$
\frac{I_{n}}{I_{m}}=\frac{A_{n} n_{n}}{A_{m} n_{m}}
$$

where $I, A$ and $n$ are intensity of spectral line, Einstein coefficients and the density of energy level. Relative intensity of spectral line $I_{n r}$ in LTE conditions can be written as following

*Corresponding Author: mtanisli@eskisehir.edu.tr

Received: 23.09.2019 Published: 16.12.2019 


$$
I_{n r}=h \frac{e^{2}}{2 \lambda^{3}} \frac{N_{0}}{Z} \frac{g_{n} f_{n r}}{\varepsilon_{0} m_{e}} \exp \left(-\frac{E_{n}}{k T_{e}}\right)
$$

The expressions in Equation 2 are as follows:

$N_{0}$ : total atomic density in the ground state,

$E_{n}$ : upper energy level,

$\varepsilon_{0}$ : permeability of free space,

$Z$ : partition function,

$g_{n}$ : statistical weight of the upper energy level,

$m_{e}$ : electron mass,

$k$ : Boltzmann constant $\left(1.38 \times 10^{-23} \mathrm{JK}^{-1}\right)$,

$e$ : electron charge,

$A_{n r}$ can be written as following

$$
A_{n r}=\frac{4 \pi e^{2}}{2 c \lambda^{2}} \frac{f_{n r}}{\varepsilon_{0} m_{e}}
$$

Then, Equation 2 can be re-arranged as Equation 4,

$$
I_{n r}=\frac{1}{4 \pi} \frac{h c N_{0}}{\lambda Z} g_{n} A_{n r} \exp \left(-\frac{E_{n}}{k T_{e}}\right)
$$

Here $r$ is the lower transition energy level which can be as the same energy level. The transitions at lower energy levels expressed by $r$ must be eliminated so that the two spectral lines are the same level [7]. If the relative intensity expressions of the two spectral lines are proportioned and arranged, the equation 5 is obtained.

$$
\frac{n_{m}}{n_{n}}=\frac{g_{m}}{g_{n}} \exp \left(-\frac{E_{m}-E_{n}}{k T_{e}}\right)
$$

When $T_{e}$ expression is obtained from Equation 5, the electron temperature can be calculated using the intensities of the two spectral lines as follows.

$$
T_{e}=\frac{E_{m}-E_{n}}{k}\left[\ln \left(\frac{A_{n} g_{n} I_{m} \lambda_{m}}{A_{m} g_{m} I_{n} \lambda_{n}}\right)\right]^{-1}
$$

\section{EXPERIMANTAL SETUP}

As shown from Figure 1, Dielectric Barrier Discharge-like (DBD-like) system at atmospheric pressure was preferred to generate plasma. The length and diameter of quartz glass were respectively $12 \mathrm{~cm}$ and $0.5 \mathrm{~cm}$ as dielectric tube in the installation of the system. $2 \%$ thorium alloy electrodes can carry higher current than other tungsten electrodes. Therefore, $2 \%$ thorium alloy tungsten electrode was used as the inner electrode and the circular aluminum electrode was used as the outer electrode. The control of the gas flow was achieved with the Mass - Stream brand M + W model D6300 series flow meter. The ELES HV-711GK5 model AC power supply, which provides a voltage range between $5-25 \mathrm{kHz}$ and a voltage between $10-20 \mathrm{kV}$, was used. Argon (Ar) gas was preferred as gas because it is an inert gas and the radiation model is simpler than other gases. Optical emission measurements for 1,2 and $3 \mathrm{~L} / \mathrm{min}$ flow rate of Ar gas with $8 \mathrm{kV}$ power and $20 \mathrm{kHz}$ frequency were made with Ocean Optics HR2000 + model OES. 


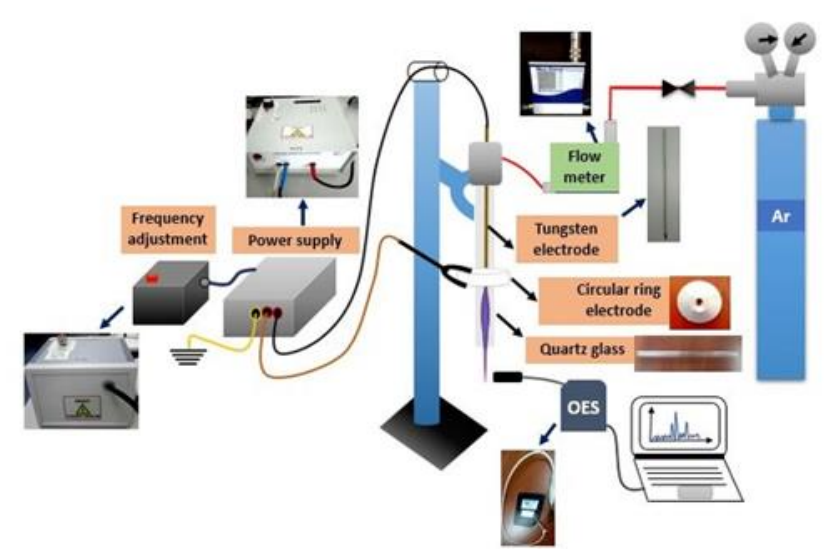

Figure 1. Schematic representation of the experimental setup

\section{RESULTS AND DISCUSSION}

DBD --like system was installed at atmospheric pressure and optical emission measurements for flow rate of Ar gas 1, 2 and $3 \mathrm{~L} / \mathrm{min}$ at $20 \mathrm{kHz}$ power were made with Ocean Optics HR2000+. As shown in in Figure 2, the intensity values corresponding to each wavelength were plotted graphically with the data obtained from the measurement results.
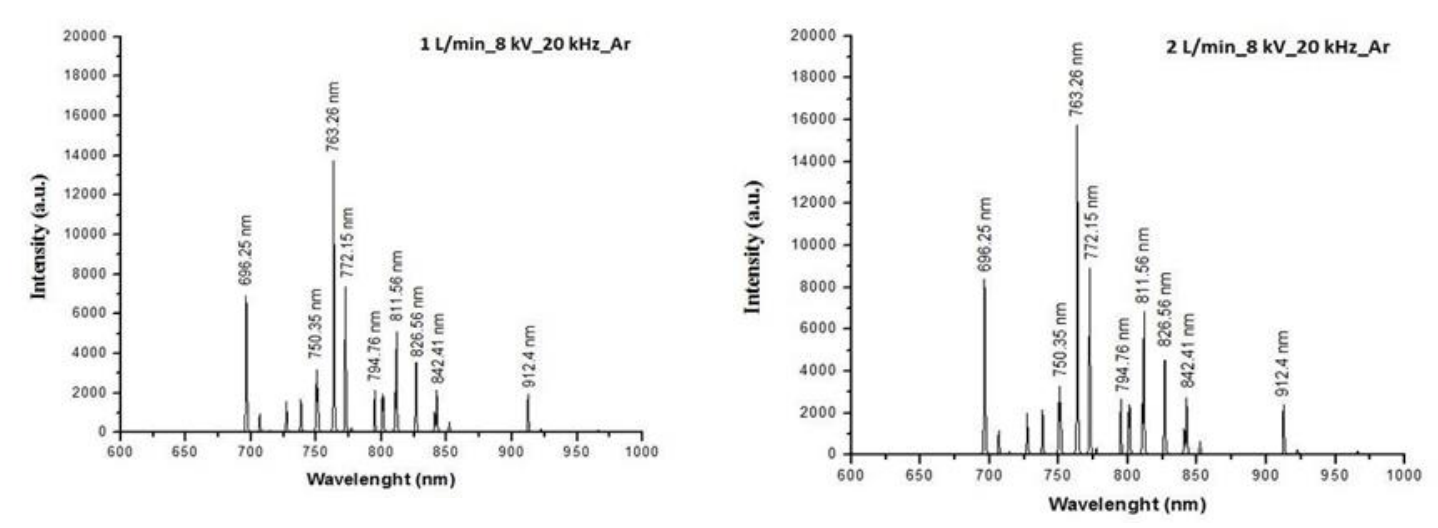

(a)

(b)

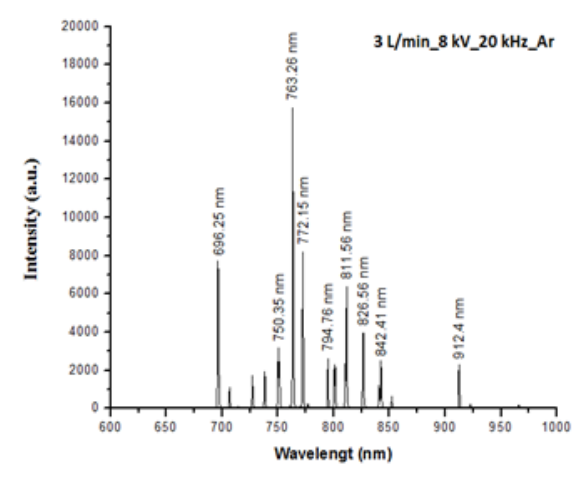

(c)

Figure 2. Spectral lines of Ar gas for (a) 1, (b) 2, (c) $3 \mathrm{~L} / \mathrm{min}$ gas flow 
For each 1, 2 and $3 \mathrm{~L} / \mathrm{min}$ flow rate of Ar gas, each peak value obtained from the results of the optical emission spectroscopy measurement is compared with the data in the NIST (National Institute of Standards and Technology) database and the wavelength value corresponding to each peak was written.

Transition probability $(A)$ values, energy values $(E)$ and statistical weights $(g)$ were obtained from the NIST database for transitions between energy levels corresponding to wavelength. The intensity values (I) were obtained from OES for 1, 2 and $3 \mathrm{~L} / \mathrm{min}$ gas flow rate. These can be seen in Tables 1 and 2.

Table 1. Intensity values and transitions at levels of 1, 2 and $3 \mathrm{~L} / \mathrm{min}$ were obtained from OES corresponding to each wavelength of Ar plasma jet [8]

\begin{tabular}{lcccc}
\hline $\boldsymbol{\lambda}(\mathbf{n m})$ & $\mathbf{1 ~ L / m i n}$ & $\begin{array}{c}\boldsymbol{I} \text { (a.u.) } \\
\mathbf{2 ~} \mathbf{\text { min }}\end{array}$ & $\mathbf{3 ~ L / m i n}$ & $\begin{array}{c}\text { Transitions } \\
\text { Lower Level- Upper Level }\end{array}$ \\
\hline 696.54 & 6931.12 & 8414.44 & 7742.03 & $4 s^{2}[3 / 2]^{0} 2-4 p^{2}[1 / 2] 1$ \\
750.38 & 3187.12 & 3315.44 & 3186.03 & $4 s^{2}[1 / 2] 1-4 p^{2}[1 / 2] 0$ \\
763.51 & 13736.12 & 15733.44 & 15738.03 & $4 s^{2}[3 / 2]^{0} 2-4 p^{2}[3 / 2] 2$ \\
772.37 & 7368.12 & 8916.44 & 8220.03 & $4 s^{2}[3 / 2]^{0} 2-4 p^{2}[1 / 2] 1$ \\
794.81 & 2163.12 & 2677.44 & 2641.03 & $4 s^{2}[1 / 2]^{0} 0-4 p^{2}[3 / 2] 1$ \\
811.53 & 5094.12 & 6869.44 & 6372.03 & $4 s^{2}[3 / 2]^{0} 2-4 p^{2}[5 / 2] 3$ \\
826.45 & 3560.12 & 4542.44 & 3971.03 & $4 s^{2}[1 / 2]^{0} 1-4 p^{2}[1 / 2] 1$ \\
842.46 & 2160.12 & 2707.44 & 2507.03 & $4 s^{2}[3 / 2]^{0} 1-4 p^{2}[5 / 2] 2$ \\
912.29 & 1981.12 & 2438.44 & 2313.03 & $4 s^{2}[3 / 2]^{0} 2-4 p^{2}[1 / 2] 1$ \\
\hline
\end{tabular}

Table 2. Transition probability values corresponding to each wavelength, energy values in transitions and statistical weight values for Ar plasma jet [8]

\begin{tabular}{ccccc}
\hline $\boldsymbol{\lambda}(\mathbf{n m})$ & $\boldsymbol{\lambda}(\mathbf{n m})(\mathbf{N I S T})$ & $\boldsymbol{A}\left(\boldsymbol{s}^{-\mathbf{1}}\right)$ & $\boldsymbol{g}_{\boldsymbol{m}}-\boldsymbol{g}_{\boldsymbol{n}}$ & $\boldsymbol{E}_{\boldsymbol{m}}-\boldsymbol{E}_{\boldsymbol{n}}(\mathbf{e V})$ \\
\hline 696.25 & 696.54 & $6.4 \times 10^{6}$ & $5-3$ & $11.55-13.33$ \\
750.35 & 750.38 & $4.5 \times 10^{7}$ & $3-1$ & $11.83-13.48$ \\
763.26 & 763.51 & $2.45 \times 10^{7}$ & $5-5$ & $11.55-13.17$ \\
772.15 & 772.37 & $5.2 \times 10^{6}$ & $5-3$ & $11.55-13.15$ \\
794.76 & 794.81 & $1.86 \times 10^{7}$ & $1-3$ & $11.72-13.28$ \\
811.56 & 811.53 & $3.3 \times 10^{7}$ & $5-7$ & $11.55-13.076$ \\
826.56 & 826.45 & $1.53 \times 10^{7}$ & $3-3$ & $11.83-13.33$ \\
842.41 & 842.46 & $2.15 \times 10^{7}$ & $3-5$ & $11.62-13.095$ \\
912.4 & 912.29 & $1.89 \times 10^{7}$ & $5-3$ & $11.55-12.91$ \\
\hline
\end{tabular}

Two spectral lines from optical emission spectroscopy measurements were selected to calculate the electron temperature of Ar plasma jet. While selecting these spectral lines, they were considered to be sensitive to electron temperature. The sensitivity of the peaks depends on the difference in excitation energy between the two transitions. The transition probability ratios of the selected peaks also affect the sensitivity of the peaks. As the energy difference increases, the sensitivity of the spectral lines is so high [9]. In order to calculate the electron temperature, energy difference and transition probability ratios of the upper levels of the spectral lines in the obtained spectrum were determined and the sensitivity spectral lines from Table 3 were selected. According to these results, the most sensitive spectral lines were determined as $763.51 \mathrm{~nm}$ and $826.45 \mathrm{~nm}$. 
Table 3. Energy difference and transition probability ratios of higher levels of spectral lines

\begin{tabular}{ccc}
\hline $\boldsymbol{\lambda}_{\mathbf{1}}-\boldsymbol{\lambda}_{\mathbf{2}}(\mathbf{n m})$ & $\Delta \boldsymbol{E}(\mathbf{e V})$ & $\boldsymbol{A}$ ratio \\
\hline $696.54-750.38$ & 0.15 & 0.142 \\
$763.51-826.45$ & 0.16 & 1.601 \\
$772.37-750.38$ & 0.33 & 0.116 \\
$811.56-842.46$ & 0.019 & 1.535 \\
$912.29-750.38$ & 0.57 & 0.420 \\
$842.46-794.81$ & 0.185 & 1.156 \\
\hline
\end{tabular}

\section{CONCLUSIONS}

In this study, the electron temperature of the plasma system generated by Ar gas at atmospheric pressure was calculated by using line intensity ratio method. The plasma system used for the experimental study with a higher density than low pressure systems was investigated. As a result of the experiments and calculations, it is concluded that the line intensity ratio method is valid for lower densities.

In the study, DBD-like plasma jet was obtained at atmospheric pressure. The electron temperature of Ar plasma jet was calculated using the intensities of the two spectral lines. Accordingly, the electron temperature values of Ar plasma jet were estimated as $0.55 \mathrm{eV}, 0.88 \mathrm{eV}$ and $4.34 \mathrm{eV}$ for 1,2 and $3 \mathrm{~L} / \mathrm{min}$ flow rates, respectively. In the literature, the electron temperature of atmospheric pressure Ar plasma jet for $2 \mathrm{~L} / \mathrm{min}$ gas flow rate was found to be $0.9 \mathrm{eV}$. When the results were compared with the literature, it was seen that the electron temperature value calculated for $2 \mathrm{~L} / \mathrm{min}$ was more compatible than the values calculated for 1 and $3 \mathrm{~L} / \mathrm{min}$

\section{ACKNOWLEDGEMENTS}

We are grateful to Eskişehir Technical University via Research Project Number: 19ADP154.

\section{REFERENCES}

[1] Tanışlı M, Mutlu MB, Poyraz N, Şahin N, Demir S. Interactions of atmospheric pressure plasma jets and microorganism: inactivation. Fresenius Environ. Bull. 2018; 27 (3): 11574-1582.

[2] Tanisli M, Mertadam S, Poyraz N, Sahin N, Demir S. Inactivation of microorganisms with neon plasma jet at atmospheric pressure. J. Pure Appl. Microbio. 2016; 10(3): 1897-1904.

[3] Tanışlı M, Taşal E, Şahin N, Arslan Ç. 6-(2-Fluorobenzoyl)-3-(2-(4-(4-fluorophenyl) piperazin-1yl)-2-oxoethyl) benzo[d]thiazol-2(3H)-one drug molecule structure and its interaction with atmospheric pressure plasma jet. Journal of Molecular Liquids 2017; 240: 733-751.

[4] Meulenbroeks RFG, Steenbakkers MFM, Qing Z, Van de Sanden MCM, Schram DC. Four ways to determine the electron density in low temperature plasmas. Phys. Rev. E 1994; 49: 2272.

[5] Lee J, Ko W, Seo D, Kim Y, Yoon J. He I line intensity ratio method for electron density and temperature measurements in multipurpose plasma (MP2). Fusion Sci. and Technol. 2009; 55 (2T): $100-105$.

[6] Zhu XM, Pu YK, Balcon N, Boswell R. Measurement of the electron density in atmosphericpressure low-temperature argon discharges by line-ratio method of optical emission spectroscopy. J. Phys. D: Appl. Phys. 2009; 42: 142003. 
[7] Zhang N, Sun F, Zhu L, Verdy C, Planche MP, Liao H, Dong C, Coddet C. Characteristics of Cu film deposited using VLPPS. J. Therm. Spray Tech. 2011; 20(1): 351-357.

[8] https://physics.nist.gov/PhysRefData/ASD/lines_form.html (Available: 08.01.2019)

[9] Boogaard A, Kovalgin AY, Aarmink AAI, Wolters RAM, Holleman J, Brunets I, Schmitz J. Measurement of electron temperatures of argon plasmas in a high-density inductively-coupled remote plasma system by Langmuir probe and optical-emission spectroscopy. Proceedings of the 9th annual workshop on Semiconductor Advances for Future Electronics and Sensors 2006; Utrecht, The Netherlands: Technology Foundation (STW), 412-418. 